decision algorithm reduced number of blood cultures without adverse events. ${ }^{10}$ Most respondents recognized that blood cultures are ordered to help with antibiotic treatment decisions. Close monitoring of broad-spectrum antibiotic use and antibiotic deescalation should occur if interventions to limit blood culture testing are implemented.

Our study was performed at a single center and we cannot exclude volunteer bias. However, there was balanced representation from the different groups surveyed and a wide range of years of experience. In summary, more specific guidance with indications for blood cultures may help reduce unnecessary blood cultures, and interventions should include all providers, including consulting physicians.

\section{Acknowledgments.}

Financial support. Support for the statistical analysis portion of this study was provided by the National Center for Research Resources and the National Center for Advancing Translational Sciences (NCATS) of the National Institutes of Health (grant no. 1UL1TR001079). No other financial support was provided relevant to this article.

Conflicts of interest. All authors report no conflict of interest relevant to this article.

\section{References}

1. Coburn B, Morris AM, Tomlinson G, Detsky AS Does this adult patient with suspected bacteremia require blood cultures? JAMA 2012;308:502-511.
2. O'Grady NP, Barie PS, Bartlett JG, et al. Guidelines for evaluation of new fever in critically ill adult patients: 2008 update from the American College of Critical Care Medicine and the Infectious Diseases Society of America. Crit Care Med 2008;36:1330-1349.

3. Riedel S, Bourbeau P, Swartz B, et al. Timing of specimen collection for blood cultures from febrile patients with bacteremia. J Clin Microbiol 2008;46:1381-1385.

4. Miller JM, Binnicker MJ, Campbell S, et al. A guide to utilization of the microbiology laboratory for diagnosis of infectious diseases: 2018 update by the Infectious Diseases Society of America and the American Society for Microbiology. Clin Infect Dis 2018. doi: 10.1093/cid/ciy381.

5. Mermel LA, Allon M, Bouza E, et al. Clinical practice guidelines for the diagnosis and management of intravascular catheter-related infection: 2009 update by the Infectious Diseases Society of America. Clin Infect Dis 2009;49:1-45.

6. Clinical and Laboratory Standards Intitute (CLSI). Principles and procedures for blood cultures; approved guideline. Document M47-A. Wayne, PA: CLSI; 2007.

7. Alahmadi YM, Aldeyab MA, McElnay JC, et al. Clinical and economic impact of contaminated blood cultures within the hospital setting. J Hosp Infect 2011;77:233-236.

8. Zwang O, Albert RK. Analysis of strategies to improve cost effectiveness of blood cultures. J Hosp Med 2006;1:272-276.

9. Eliakim-Raz N, Bates DW, Leibovici L. Predicting bacteraemia in validated models—a systematic review. Clin Microbiol Infect 2015;21:295-301.

10. Woods-Hill CZ, Fackler J, Nelson McMillan K, et al. Association of a clinical practice guideline with blood culture use in critically ill children. JAMA Pediatr 2017;171:157-164.

\title{
An electronic antimicrobial stewardship intervention reduces inappropriate parenteral antibiotic therapy
}

\author{
Sean T. H. Liu MD, PhD, Mark J. Bailey ${ }^{1, a}$, Allen Zheng MS ${ }^{1, a}$, Patricia Saunders-Hao PharmD², \\ Adel Bassily-Marcus $\mathrm{MD}^{3}$, Maureen Harding $\mathrm{MSN}^{1}$, Meenakshi Rana $\mathrm{MD}^{1}$, Roopa Kohli-Seth $\mathrm{MD}^{3}$, Gopi Patel MD ${ }^{1}$, \\ Shirish Huprikar MD ${ }^{1}$ and Talia H. Swartz MD, $\mathrm{PhD}^{1}$ \\ ${ }^{1}$ Department of Medicine at the Icahn School of Medicine at Mount Sinai, New York, New York, ${ }^{2}$ Department of Pharmacy, Mount Sinai Hospital, New York, \\ New York and ${ }^{3}$ Department of Surgery, Icahn School of Medicine at Mount Sinai, New York, New York
}

To the Editor-A peripherally inserted central catheter (PICC) provides outpatient access to long-term parenteral antibiotics. ${ }^{1,2}$ Most antimicrobial stewardship systems do not have an outpatient parenteral antibiotic therapy (OPAT) program or mandatory infectious diseases (ID) consultation prior to patient discharge. ${ }^{3}$ Such programs ensure that PICC insertions are appropriate to avoid excessive antibiotic usage, line-associated complications, and antimicrobial resistance. ${ }^{4-6}$ Here, we report a simple intervention that identifies patients at risk for unnecessary parenteral antibiotics and recommends ID consultation.

Author for correspondence: Talia Swartz MD, Department of Medicine at the Icahn School of Medicine at Mount Sinai, New York, New York, 1 Gustave L. Levy Place, Box 1090, NY, New York 10029. E-mail: talia.swartz@mssm.edu

a Authors of equal contribution.

Cite this article: Liu STH, et al. (2018). An electronic antimicrobial stewardship intervention reduces inappropriate parenteral antibiotic therapy. Infection Control \& Hospital Epidemiology 2018, 39, 1396-1397. doi: 10.1017/ice.2018.220
The intervention resulted in an $84 \%$ relative risk reduction in patients discharged with PICC insertions without ID consultation.

The study was performed at a 1,134-bed academic medical center. Between January 2016 and May 2016, 181 PICC orders were placed for parenteral antibiotics (Fig. 1A), and 172 of these included ID consultation (95\%) while 9 (5\%) did not. Two ID attending physicians retrospectively reviewed these 9 cases and concluded that 8 PICC insertions (89\%) were inappropriate.

An electronic intervention was implemented into the PICC insertion order set in November 2016. Three prompts were inserted to ensure ID consultation for all PICC insertions for parenteral antibiotics: (1) "Will the PICC be used for IV antibiotics?" A "No" response prompted the original order set. A "Yes" prompted the following question: (2) "Is ID consulted?" A "No" prompted the statement: "ID consultation is required 
A

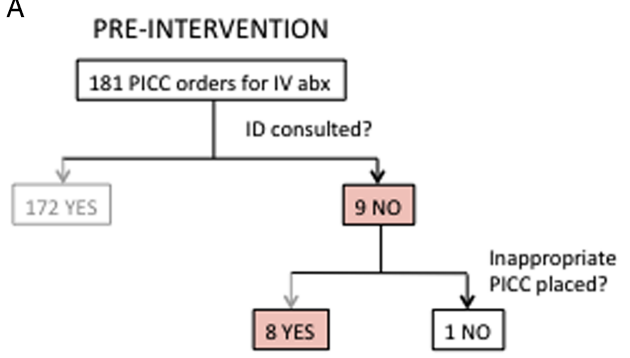

B

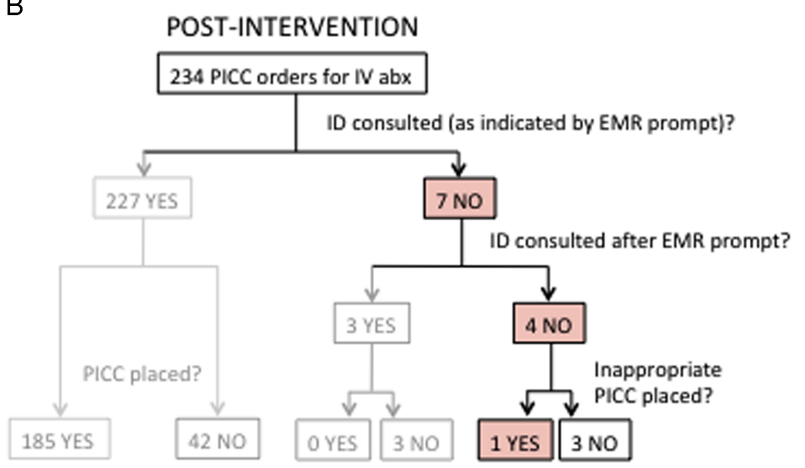

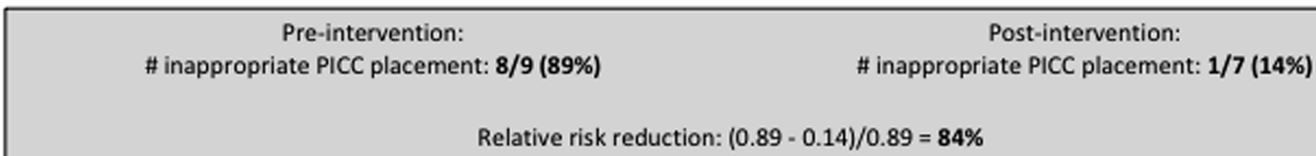

Fig. 1. Pre- and postintervention flowchart. (A) Before the intervention, 181 PICC insertion orders were placed for parenteral antibiotics. Overall, 172 patients received ID consultation, while 9 patients did not, and 8 of those 9 patients had an inappropriate PICC insertions. (B) Postintervention, 234 orders for PICC insertions were placed for parenteral antibiotics. Among 227 patients indicating that ID was consulted, 185 patients had PICC placement and 42 did not. Seven patients were prompted to consult ID. For 3 of those patients, providers consulted ID and the patients ultimately did not have PICC insertions. For 4 of those patients, ID consultation was not obtained. Of those 4 patients, 3 did not require a PICC, while 1 had a PICC placed that was deemed inappropriate.

prior to PICC placement for IV antibiotics. Please consult an ID team." (3) In this case, the provider would need to select: "ID will be consulted immediately." This project was a quality improvement initiative conducted by the Quality Improvement Committee at the Mount Sinai Hospital.

After launching the electronic intervention, 234 PICC orders were placed for parenteral antibiotics between December 2016 to May 2017 (Figure 1B). Seven instances triggered "ID will be consulted immediately." Three patients had ID consultation and did not have a PICC inserted. Four patients did not have ID consultation. Among these, 3 patients were switched to oral antibiotics without ID consultation. Finally, 1 patient (among the 7 instances) was discharged with a PICC insertion without ID consultation and had an unfavorable outcome.

1 of 7 patients was discharged with an inappropriate PICC insertion without an ID consultation, a reduction of $89 \%$ (8 of 9 patients) before the intervention. Thus, the EMR intervention resulted in an $84 \%$ relative risk reduction of inappropriate PICC insertions. All charts for whom PICC insertions occurred without ID consultation were independently reviewed by 2 ID attending physicians. In 24 of 26 reviews (92\%), ID consultation would have changed treatment management to avoid PICC insertion.

In conclusion, this intervention provides a sustainable and cost-effective antimicrobial stewardship approach to reduce inappropriate parenteral antibiotics usage. It also highlights the importance of ID consultation in determining the appropriateness of long-term outpatient parenteral antibiotic therapy.
Acknowledgements. We thank the EMR team for implementing the electronic intervention.

Financial support. This work was supported by a grant from the National Institutes of Health (NIH)/National Institute of Allergy and Infectious Diseases (NIAID) (grant no. K-08AI120806).

Conflicts of interest. All authors report no conflicts of interest relevant to this article.

\section{References}

1. Wai AO, Frighetto L, Marra CA, Chan E, Jewesson PJ. Cost analysis of an adult outpatient parenteral antibiotic therapy (OPAT) programme. A Canadian teaching hospital and Ministry of Health perspective. PharmacoEconomics 2000;18:451-457.

2. Goodfellow AF, Wai AO, Frighetto L, et al. Quality-of-life assessment in an outpatient parenteral antibiotic program. Ann Pharmacother 2002;36:1851-1855.

3. Lane MA, Marschall J, Beekmann SE, et al. Outpatient parenteral antimicrobial therapy practices among adult infectious disease physicians. Infect Control Hosp Epidemiol 2014;35:839-844.

4. Shrestha NK, Bhaskaran A, Scalera NM, Schmitt SK, Rehm SJ, Gordon SM. Antimicrobial stewardship at transition of care from hospital to community. Infect Control Hosp Epidemiol 2012;33:401-404.

5. Tice AD, Rehm SJ, Dalovisio JR, et al. Practice guidelines for outpatient parenteral antimicrobial therapy. IDSA guidelines. Clin Infect Dis 2004;38:1651-1672.

6. Paladino JA, Poretz D. Outpatient parenteral antimicrobial therapy today. Clin Infect Dis 2010;51 Suppl 2:S198-S208. 